\title{
Three-block electrical model of renal impedance
}

\author{
Tse Lin Hsu ${ }^{1}$, Hsin Hsiu ${ }^{2}$, Pin Tsun Chao ${ }^{3}$, Sai Ping Li ${ }^{1}$, Wei Kung Wang ${ }^{1}$ \\ and Yuh Ying Lin Wang ${ }^{1,4}$ \\ ${ }^{1}$ Biophysics Laboratory, Institute of Physics, Academia Sinica, Nankang, Taipei 11529, \\ Taiwan, Republic of China \\ 2 Department of Electrical Engineering, Yuan Ze University, Taoyuan, Taiwan, Republic of China \\ ${ }^{3}$ Electrical Engineering Department, National Taiwan University, Taipei, Taiwan, \\ Republic of China \\ ${ }^{4}$ Physics Department, National Taiwan Normal University, Taipei, Taiwan, Republic of China \\ E-mail: linhsu@phys.sinica.edu.tw,hhsiu@saturn.yzu.edu.tw,pt_chao@pchome.com.tw, \\ spli@phys.sinica.edu.tw, wkwang@phy.sinica.edu.tw and yuhying@phy.ntnu.edu.tw
}

Received 15 December 2004, accepted for publication 14 March 2005

Published 4 April 2005

Online at stacks.iop.org/PM/26/387

\begin{abstract}
In this study, we measured the characteristic renal impedance profiles of Wistar rats and simulated the profiles using an electrical model with three series connected Windkessel blocks containing inductance. It is expected that a complete renal impedance profile ought to provide better physical properties information and have more diagnostic power than the pulsatility (PI) and resistive indices (RI) as a result of frequency dependency. A characteristic peak value at the third harmonic on the renal impedance amplitude curve was observed and the phase curve decreased with increasing harmonic numbers. From least mean square fitted parameters, the three blocks were given distinct physical properties and identified as: (1) the renal artery, (2) the small arteries plus the afferent arteriole and (3) the residual kidney (i.e., the efferent arteriole plus the post glomerular capillary structures). These allocations were made according to respective physical properties reported in previous research. These classifications were further confirmed when we compressed the kidney or infused Ang II. Variations in electrical parameters concurred with the likely affected blood vessels reported. This model describes renal impedance characteristics well; and it provides useful hints on the physical properties of the renal vascular system as well as allows for distinctions in possible physiologically affected locations during functional disturbance. It has potential for development as a clinical non-invasive diagnostic tool.
\end{abstract}

Keywords: renal impedance, electrical model, angiotensin II, compression effect, least mean square fitting 


\section{Introduction}

\subsection{Motivation for studying the complete renal impedance spectrum}

One of the most important issues in hemodynamics is to resolve the relationship between pressure and flow in terms of physical properties of the cardiovascular system under varying conditions such as drugs, hypertension and so on. In clinical renal vascular studies, the pulsatility index (PI) (Gosling and King 1974) and the resistive index (RI) are both calculated from the averaged blood pressure and flow values obtained from a Doppler sonograph. These indices have proved to be helpful in many clinical situations (Handa et al 1986, Veglio et al 1995, Karadeniz et al 1996) but they have their limitations (Merkus et al 1994, Salgado et al 1997, Pozniak et al 1988, Warshauer et al 1988). It is expected that a complete renal impedance spectrum with frequency dependency ought to provide a good deal more information about the physical properties of the renal vascular bed and hence offer greater diagnostic power than both the PI and RI.

\subsection{Advancement in impedance models}

Because relations between electrical voltage $(V)$ and electrical current $(I)$ are analogous to the relations between blood pressure $(P)$ and blood flow $(Q)$, electrical models constructed with basic electric elements (electrical resistance $R$, inductance $L$ and capacitance $C$ ) are extensively used to simulate the physical properties of blood vessels (hemodynamic resistance $R_{\text {vas }}$, inertance $L_{\text {vas }}$ and compliance $C_{\text {vas }}$ ) (Milnor 1989). A proper electrical model may provide ready formulas to analyze the impedance spectrum and therefore may illustrate the 'collective' or 'macroscopic' physical characteristics of the organ or vascular bed (Mo et al 1988, Noordergraaf 1969, Raines et al 1974).

There are several models that have proved successful in illustrating impedance profiles of different vascular beds. Mo et al (1988) built an electrical model to simulate the umbilicoplacental circulation. It was promising in predicting diameter change, and incident and reflected components of both arterial pressure and flow wave forms. The model consisted of a uniform transmission line that represented the artery and load impedance (with a resistor and a capacitor in parallel) that represented the vascular bed. Hill et al (1995) modified Mo's model into a viscoelastic (inductance added) two-load model and got a better fit for the impedance phase and the measurements following placental embolization and during angiotensin II infusion. Czosnyka et al (1994) used a circuit containing inductance to simulate the cerebral circulation of rabbits. Giller et al (1996) successfully analyzed the impedance index of human cerebral circulation in normal subjects and in subarachnoid hemorrhage with a model comprising two Windkessels connected in series, which represented the large vessel and the cerebrovascular bed respectively. It was found that they could yield a peak curve as observed in the case of vasospasm only if an inductance element was included in the large vessel component. These models indicate that multiple loads may refine fittings, and inductance is necessary to achieve the characteristic peak curve.

\subsection{Previous renal impedance studies}

However, for the kidneys, which play an important role in regulating blood pressure and maintaining the proper physiological condition of the body, there have been few attempts at resolving renal impedance spectrum profiles resulting in a limited number of models ever being studied (Spencer and Denison 1963, Rothe and Nash 1967). In these few early reports, the kidney was treated as a single entity and only the relationships between the averaging pressure 
and flow were discussed. The problem lies with the fact that renal vasculature contains distinct functional sub-vessel groups (such as glomerulus) which are physiologically important. If we treat the kidney as a single entity, we may get blurred 'collective' physical characteristics of the kidney as shown in those previous reports, but lose a lot of the important physiological information from individual vessel groups and thereby negate much of the clinical or academic usefulness. A better idea would be to classify the functional groups and model them as different linked electrical blocks with specified physical characteristics of their own. For models with a small number of blocks, there are ready formulas for impedance calculation and the timing for analysis is endurable.

\subsection{Our approach to renal impedance studies}

In this report, we constructed a simple electrical model with three linked blocks containing only a few electrical elements and then we tried to find the parameters of best fit to simulate the impedance profiles measured. Models from Czosnyka et al (1994), Giller et al (1996) and Hill et al (1995) were referenced; we decomposed the renal artery and the renal vascular bed into three blocks and inductance was added to give the characteristic peaks on the impedance amplitude curve.

The fidelity of this best-fitted model was further checked by compressing rat kidneys and infusion with angiotensin II (Ang II) to see if the affected locations of the renal vascular system could be identified from the varied electrical parameters of the corresponding blocks.

\section{Materials and methods}

\subsection{Animal preparation}

Male Wistar rats weighing between $240 \mathrm{~g}$ and $320 \mathrm{~g}$ were obtained from the Experimental Animal Center of National Taiwan University, Taipei, Taiwan. The animals were housed in our animal care facility with a 12-h light/dark cycle; they were allowed water ad libitum and food (Labdiet 5001 Rodent diet, PMI Nutrition International LLC, Brentwood, MO, USA) prior to the experiments.

The set-up for the experiment is shown in figure 1. The rats were anesthetized with urethane $\left(1.1 \mathrm{~g} \mathrm{~kg}^{-1}\right.$, intraperitoneal). We measured the pressure wave in the abdominal aorta and flow wave in the renal artery. Cannulation from the root of the tail artery (arteria caudalis) was as close as possible to the entrance of the renal artery with an intravenous catheter (Angiocath Plus, $22 \mathrm{GA}, 1.00 \mathrm{IN}, 0.9 \times 25 \mathrm{~mm}^{2}$, Becton-Dickinson, Korea) filled with physiological saline and heparin, which was then connected to a pressure transducer (P10EZ Ohmeda (s) Pte. Ltd., Singapore). The left renal artery (a. renalis) was reached from the dorsal side of the rat's body. The volumetric flow of the renal artery was converted from the measured voltage by an ultrasonic pulsed Doppler flow system (Crystal Biotech VF-1, PD-20 module; $62.5 \mathrm{kHz}$ pulse repetition frequency, $20 \mathrm{Mhz}$, and $0.8 \mathrm{~mm}$ probe).

The femoral vein was exposed through an inguinal incision and a polyethylene cannula (PE-10) was inserted into it for Ang II injection.

\subsection{Recording procedure}

Both the pressure and flow wave signals were sent to a preamplifier (Universal amplifier, Gould Instrument Systems, Inc. Ohio, USA) and then to a 16 bit A/D converter AX5621 (Axiom technology Co. LTD, Taiwan, ROC) that was an interface card for the personal computer used in data analysis. 


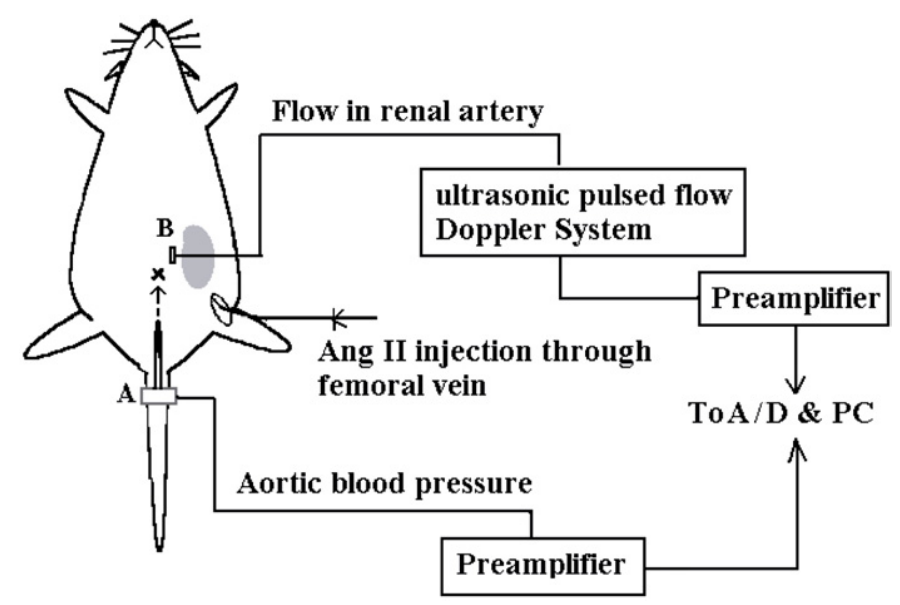

Figure 1. The set-up for the renal impedance study experiment is shown. The pressure wave in the abdominal aorta was measured from the root of the tail artery through an intravenous catheter which was connected to a pressure transducer (A). The left renal artery was reached from the dorsal side of the rat's body and its flow wave was measured by a $0.8 \mathrm{~mm}$ ultrasonic pulsed Doppler flow probe (B). Both the pressure and flow wave signals were sent to a preamplifier and then to a 16 bit A/D converter for the personal computer used in data analysis. For group B, the femoral vein was exposed and a polyethylene cannula (PE-10) was inserted into it for Ang II injection.

Rats were divided into two groups: group A (16 rats) for kidney compressing, and group B (34 rats) for Ang II injection (7.5 ng/30 ul saline). After all surgical operations were completed, the rat was fed $1 \mathrm{ml}$ water and then put to rest for at least $120 \mathrm{~min}$ until its blood pressure and heart rate stabilized; over this period the kidney surface was kept moist using occasional drops of saline. The experiment then proceeded as follows:

1. Half hour self-controlled data were taken. One-second length data sequence (5-8 pulses included) was systematically sampled every minute. A total of 30 data sequences (about 200 pulses) were recorded in half an hour as control data. Three criteria were employed to ensure the control data sampling was sufficiently accurate, representative and stable. The $\mathrm{CV}$ (SD/mean) for the heart rate, the diastolic pressure and the amplitudes of the first two harmonics of the pressure wave were all required to be less than 5\%. An animal would be considered to be non-stable and data would be discarded if the CV values exceeded this limit.

2. After the control period, each group of rats was treated as follows:

Group A: the rat's kidney was compressed by putting a $150 \mathrm{~g}, 0.75 \mathrm{~cm}$ diameter copper bar on the kidney surface for $1 \mathrm{~min}$, and the compression effect was recorded continuously from the beginning to the end of stimulation (a total of 60 one-second data sequences). The copper bar was bathed in hot water to keep it at $37^{\circ} \mathrm{C}$.

Group B: Ang II (7.5 ng/30 ul saline) was infused via the femoral vein within $3 \mathrm{~s}$. The effects of the drug were recorded continuously for 1 min after beginning the infusion (a total of 60 one-second data sequences).

\subsection{Data analysis}

Individual pulses were separated from the less noisy pressure wave sequence at its two lowest points (the place where two neighboring systolic pressures started). Any pressure pulse with 
a difference between the two lowest points larger than $1 \%$ was rejected. The same timing points for qualified pressure pulses were used to separate the flow pulses on the corresponding flow wave sequence. Each isolated pressure-flow pulse pair was then transformed into the frequency domain with a discrete Fourier transformation (DFT). No filtering, windowing or trending was used in this transforming process.

For each selected pulse, the mean value (the dc component) $A_{\mathrm{dc}}$ as well as the amplitude $A[n]$ and phase angle $\theta[n]$ was calculated, where $n$ is the corresponding harmonic. Since the amplitudes decreased rapidly with the harmonic numbers, we focused our attention only on the first seven harmonics, that is, $n=1-7$.

The amplitudes of harmonics $1-7$ of the pressure spectrum were divided by the amplitude of the corresponding harmonics of the flow spectrum to get the amplitude of the impedance $A_{\mathrm{Z}}[n]=\frac{A_{\text {pressure }}[n]}{A_{\text {fow }}[n]}$, and the impedance phase was calculated as the phase difference between the pressure and flow for each harmonic $\theta_{Z}[n]=\theta_{\text {pressure }}[n]-\theta_{\text {flow }}[n]$. Here, phase will be negative if the flow wave leads the pressure wave. The renal vascular resistance is defined as $\operatorname{RVR}=\frac{A_{\text {dc,pressure }}}{A_{\text {dc.flow }}}$.

For each experimental stage, the renal impedance of a rat was the average of all its recorded pressure-flow pulse pairs. The final averaged impedance profiles were acquired through the total averaging of all the rats in each group.

The compressed kidney effect and infusion Ang II effect were calculated as the percentage of the difference in amplitude as well as the difference in phase angle between the treatments and the control.

\subsection{Statistical analysis}

A paired- $t$ test was used for statistically deciding characteristic points (the impedance minimal, maximum) of the impedance profiles. Each point on the impedance profiles was compared with its neighboring points; it was considered a characteristic impedance minimal (or maximum) point if its value was significantly less (or more) than the values of both its neighboring points as ${ }^{*} p<0.05$.

To find the best-fitted model, we first varied the parameters of a model to reach the least mean square difference between the amplitudes of the calculated and the experimental impedance for harmonics 1-7. Then the characteristic points of the theoretical impedance profiles (amplitude and phase) where the natural frequencies were located were checked; only the model with the appropriately located characteristic points was considered to be the right best-fitted model otherwise the model was abandoned.

\subsection{Electrical model}

In this report, the three-Windkessel model as shown in figure 2 was used to simulate the renal vascular system. In each block of the model, the resistance $\left(R_{\mathrm{a} 1}, R_{\mathrm{a} 2}, R_{\mathrm{r}}\right)$ was series connected with inductance $\left(L_{\mathrm{a} 1}, L_{\mathrm{a} 2}, L_{\mathrm{r}}\right)$ and then parallel connected with capacitance $\left(C_{\mathrm{a} 1}\right.$, $C_{\mathrm{a} 2}, C_{\mathrm{r}}$ ). The physical properties of the renal artery, the small arteries plus the afferent arteriole and the residual of the kidney (the efferent arteriole plus the post glomerular capillary structures mainly) were assumed to be responsible for $\left(R_{\mathrm{a} 1}, L_{\mathrm{a} 1}, C_{\mathrm{a} 1}\right),\left(R_{\mathrm{a} 2}, L_{\mathrm{a} 2}, C_{\mathrm{a} 2}\right)$ and $\left(R_{\mathrm{r}}, L_{\mathrm{r}}, C_{\mathrm{r}}\right)$ respectively. A similar two-load model was proved to be helpful in simulating the umbilicoplacental circulation by Hill et al (1995).

For sinusoidal currents and voltages, the impedance equations for the model in figure 2 expressed with complex variables are given in the appendix. The equations are expressed in radial frequency $\omega=2 \pi f$, where $f$ is the frequency in $\mathrm{Hz}$. 


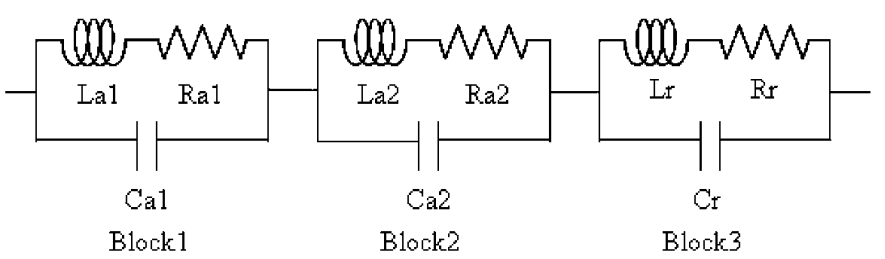

Figure 2. The three-block electrical model used for renal vascular impedance simulation. The impedance equations of the model are described in the appendix. The renal vascular system was divided into the renal artery part $\left(R_{\mathrm{a} 1}, L_{\mathrm{a} 1}, C_{\mathrm{a} 1}\right)$, the small arteries plus the afferent arteriole part $\left(R_{\mathrm{a} 2}, L_{\mathrm{a} 2}, C_{\mathrm{a} 2}\right)$, and the residual part of the kidney $\left(R_{\mathrm{r}}, L_{\mathrm{r}}, C_{\mathrm{r}}\right)$. The electrical resistance $R_{\mathrm{a} 1}, R_{\mathrm{a} 2}, R_{\mathrm{r}}$, inductance $L_{\mathrm{a} 1}, L_{\mathrm{a} 2}, L_{\mathrm{r}}$ and capacitance $C_{\mathrm{a} 1}, C_{\mathrm{a} 2}, C_{\mathrm{r}}$ are equivalent to the hemodynamic resistance, inertance and compliance of each of the divided parts.

Table 1. Physiological data, the diastolic pressure (DP), systolic pressure (SP), mean blood pressure (AVEP), heart rate (HR), renal blood flow (RBF) and renal vascular resistance (RVR) of both groups A and B are presented. The compressed kidney effect as well as the Ang II effect on these physiological parameters are also shown.

\begin{tabular}{lllllll}
\hline & $\begin{array}{l}\mathrm{DP} \\
(\mathrm{mmHg})\end{array}$ & $\begin{array}{l}\mathrm{SP} \\
(\mathrm{mmHg})\end{array}$ & $\begin{array}{l}\text { AVEP } \\
(\mathrm{mmHg})\end{array}$ & $\mathrm{HR}(\mathrm{Hz})$ & $\begin{array}{l}\mathrm{RBF} \\
\left(\mathrm{ml} \mathrm{min}^{-1}\right)\end{array}$ & $\begin{array}{l}\mathrm{RVR} \\
\left(10^{5} \mathrm{dyn} \mathrm{s} \mathrm{cm}^{-5}\right)\end{array}$ \\
\hline Group A & & & & & & \\
Control $\pm \mathrm{SE}$ & $71.48 \pm 1.75$ & $118.71 \pm 2.25$ & $90.19 \pm 1.82$ & $6.95 \pm 0.13$ & $4.92 \pm 0.21$ & $14.72 \pm 0.64$ \\
Compressing & $77.09 \pm 1.59$ & $124.90 \pm 2.18$ & $95.97 \pm 1.63$ & $7.07 \pm 0.12$ & $3.91 \pm 0.31$ & $21.05 \pm 1.63$ \\
& $* * *$ & $* * *$ & $* * *$ & $* * *$ & $* * *$ & $* * *$ \\
Group B & & & & & & $14.63 \pm 0.39$ \\
Control $\pm \mathrm{SE}$ & $71.00 \pm 1.25$ & $115.98 \pm 1.17$ & $88.87 \pm 1.12$ & $6.87 \pm 0.08$ & $4.81 \pm 0.09$ & $14.52 \pm 0.55$ \\
Ang II & $76.15 \pm 1.32$ & $120.394 \pm 1.15$ & $93.84 \pm 1.14$ & $6.67 \pm 0.08$ & $4.32 \pm 0.08$ & $17.52 \pm 0$ \\
& $* * *$ & $* * *$ & $* * *$ & $* * *$ & $* * *$ & $* * *$ \\
\hline
\end{tabular}

SE: Standard error.

${ }^{* * *} p<0.0001$ : The probability that the experimental effect is significantly different from the control value.

For a periodical signal as the pressure pulse, most of its energy is focused on its harmonic bands (Jan et al 2000), so we considered the impedance at the harmonic frequency $f_{n}=n f_{0}$ only, in which $f_{0}$ is the heart rate, in which $\omega=2 \pi n f_{0}=n \omega_{0}$.

\section{Results}

\subsection{Basic physiological information}

Table 1 provides the basic physiological information for both groups. The control values for both groups were similar and stable. Compressing the kidney or infusion of Ang II caused small but significant changes to all these indices; both stimuli increased the blood pressure, decreased renal blood flow and as a consequence increased renal vascular resistance. However the heart rate varied in opposite directions, in that we observed an increased heart rate during kidney compressing, and a decreased rate post Ang II infusion.

\subsection{Renal impedance spectra}

The impedance spectra measured for groups A and B are given in tables 2 and 3, respectively. Both groups had characteristically maximum impedance points at the third harmonic and they 
Table 2. The renal impedance spectra for group A are presented. The compressed kidney effects are expressed as the percentage of the difference in amplitude (\%Diff. Amp.) and the difference in phase angle (Diff. Phase) between the control and the compressing periods.

\begin{tabular}{|c|c|c|c|c|c|c|}
\hline \multirow[b]{2}{*}{ Harmonic no. } & \multicolumn{3}{|c|}{ Amplitude $\pm \mathrm{SE}\left(\times 10^{5} \mathrm{dyn} \mathrm{s} \mathrm{cm}^{-5}\right)$} & \multicolumn{3}{|c|}{ Phase $\pm \operatorname{SE~}\left({ }^{\circ}\right)$} \\
\hline & Control & Compressing & \%Diff. Amp. & Control & Compressing & Diff. Phase \\
\hline 0 & $14.72 \pm 0.64^{\mathrm{a}}$ & $21.05 \pm 1.63^{\mathrm{a}}$ & $41.13^{* * *}$ & & & \\
\hline 1 & $8.45 \pm 0.33^{\mathrm{a}}$ & $10.29 \pm 0.82^{\mathrm{a}}$ & $21.09^{* *}$ & $-6.5 \pm 2.2$ & $-10.0 \pm 2.4$ & $-3.43^{*}$ \\
\hline 2 & $10.55 \pm 0.54$ & $12.30 \pm 1.21$ & $15.92^{*}$ & $-14.1 \pm 3.4$ & $-16.80 \pm 3.2$ & -2.67 \\
\hline 3 & $18.01 \pm 1.13^{\mathrm{a}}$ & $19.18 \pm 1.76^{\mathrm{a}}$ & 7.83 & $-39.1 \pm 6.5$ & $-40.84 \pm 4.7$ & -1.74 \\
\hline 4 & $13.51 \pm 0.77$ & $15.65 \pm 1.64$ & 15.94 & $-86.9 \pm 4.8$ & $-86.78 \pm 4.4$ & 0.12 \\
\hline 5 & $13.80 \pm 0.71$ & $15.44 \pm 1.29$ & 11.71 & $-99.1 \pm 6.4$ & $-98.17 \pm 6.0$ & 0.89 \\
\hline 6 & $14.74 \pm 0.72$ & $15.89 \pm 1.41$ & 7.73 & $-106.5 \pm 12.0$ & $-116.83 \pm 7.7$ & -10.29 \\
\hline 7 & $11.97 \pm 0.90$ & $13.22 \pm 1.76$ & 10.58 & $-129.8 \pm 13.7$ & $-148.16 \pm 8.2$ & -18.34 \\
\hline
\end{tabular}

a The characteristic impedance minimal or maximum point, if its value is significantly less (or more) than the values of both its neighboring points $p<0.05$.

${ }^{*} p<0.05,{ }^{* *} p<0.001,{ }^{* *} p<0.0001$ : The probability that the compressed kidney effect is significantly different from the control value.

SE: Standard error.

Table 3. The renal impedance spectra for group B are presented. The Ang II effects are expressed as the percentage of the difference in amplitude (\%Diff. Amp.) and the difference in phase angle (Diff. Phase) between the control and the after drug (Ang II) periods.

\begin{tabular}{|c|c|c|c|c|c|c|}
\hline \multirow[b]{2}{*}{ Harmonic no. } & \multicolumn{3}{|c|}{ Amplitude $\pm \mathrm{SE}\left(\times 10^{5} \mathrm{dyn} \mathrm{scm}^{-5}\right)$} & \multicolumn{3}{|c|}{ Phase $\pm \mathrm{SE}\left({ }^{\circ}\right)$} \\
\hline & Control & Ang II & \%Diff. Amp. & Control & Ang II & Diff. Phase \\
\hline 0 & $14.63 \pm 0.36^{\mathrm{a}}$ & $17.52 \pm 0.55^{\mathrm{a}}$ & $19.76^{* * *}$ & & & \\
\hline 1 & $7.41 \pm 0.27$ & $8.43 \pm 0.27$ & $13.76^{* * *}$ & $-34.8 \pm 1.3$ & $-35.10 \pm 1.1$ & -0.26 \\
\hline 2 & $7.07 \pm 0.30^{\mathrm{a}}$ & $7.81 \pm 0.28^{\mathrm{a}}$ & $10.42^{* * *}$ & $-57.5 \pm 1.9$ & $-55.80 \pm 1.6$ & $1.26^{*}$ \\
\hline 3 & $8.60 \pm 0.40^{\mathrm{a}}$ & $9.14 \pm 0.38^{\mathrm{a}}$ & $6.30^{* *}$ & $-88.6 \pm 3.5$ & $-84.56 \pm 3.1$ & $4.04^{* *}$ \\
\hline 4 & $6.01 \pm 0.34$ & $6.84 \pm 0.38$ & $13.83^{* * *}$ & $-124.1 \pm 4.4$ & $-117.12 \pm 4.2$ & $6.94^{* * *}$ \\
\hline 5 & $5.44 \pm 0.33$ & $5.70 \pm 0.32$ & $4.76^{*}$ & $-138.9 \pm 4.7$ & $-136.16 \pm 3.7$ & 2.73 \\
\hline 6 & $5.43 \pm 0.34$ & $5.43 \pm 0.28$ & -0.55 & $-142.9 \pm 6.4$ & $-146.02 \pm 5.1$ & -3.06 \\
\hline 7 & $4.07 \pm 0.27$ & $4.27 \pm 0.25$ & $5.02^{*}$ & $-125.2 \pm 7.4$ & $-139.96 \pm 5.9$ & $-14.75^{* * *}$ \\
\hline
\end{tabular}

a The characteristic impedance minimal or maximum point, if its value is significantly less (or more) than the values of both its neighboring points $p<0.05$.

${ }^{*} p<0.05,{ }^{* *} p<0.001,{ }^{* * *} p<0.0001$ : The probability that the Ang II effect is significantly different from the control value.

SE: Standard error.

had the same level of dc resistances. There is a characteristic minimum point at the first harmonic for group A, but the minimum point is at the second harmonic for group B.

Compressing the kidney or infusion of Ang II increased the renal impedance for most of the harmonics. The increases were statistically significant for the dc and the first two harmonics when the kidney was compressed; however, after Ang II infusion, there were significant effects for $\mathrm{dc}$ and all the harmonics except the sixth harmonic. Tables 2 and 3 also present the phase profiles. The negative phase angles on all harmonics indicate that the flow wave leads the pressure. Compressing the kidney caused significant phase change at the first harmonic; but infusion with Ang II caused significant phase changes from the second to the fourth harmonics. 


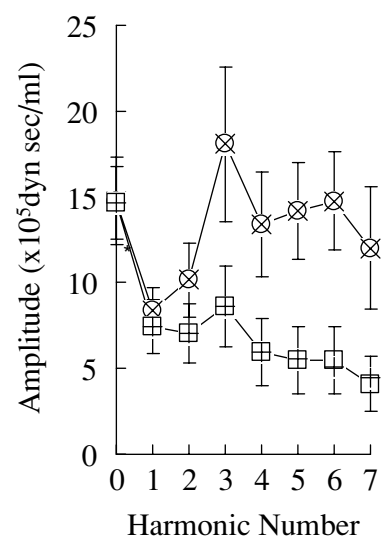

$\times$ calculated $\mathrm{A}-\mathrm{O}$ measured $\mathrm{A}$
$+\quad$ calculated $\mathrm{B}-$ measured $\mathrm{B}$

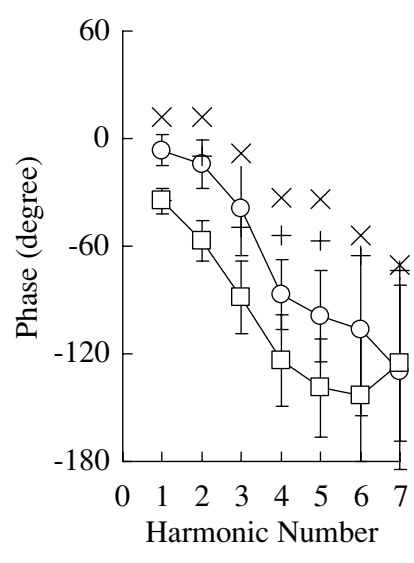

$X$ calculated $\mathrm{A}-\mathrm{O}$ - measured $\mathrm{A}$

+ calculated B $-\square-$ measured B

Figure 3. The measured renal impedance profiles of the controls for both groups A and B were plotted (measured A and measured B). The left shows the amplitude profiles and the right shows the phase profiles. The standard deviations are given as the vertical bars. The profiles were compared with their least mean square fitted results (calculated A and calculated B) calculated from the impedance equations described in the appendix.

\subsection{Least mean square fitted curves}

The control renal impedance profiles measured for both groups as well as the least mean square fitted impedance profiles calculated from the impedance equations described in the appendix are given in figure 3. The least mean square fitted amplitude profiles nearly perfectly match the experimental ones and the calculated phase profiles also show a similar trend to the measured curves. However, the calculated phase profiles have smaller negative phase values than the measured curves. For each harmonic, the difference is about $20^{\circ}$. There is about $7 \mathrm{~cm}$ of extra travel distance for the pressure wave to go from the measuring point (the intravenous catheter tip) to the pressure transducer; a $20^{\circ}$ delay is a reasonable value for an animal with a heart rate at $7 \mathrm{~Hz}$ and a pressure wave velocity of $10 \mathrm{~m} \mathrm{~s}^{-1} .7 \mathrm{~cm} /\left(10 \mathrm{~m} \mathrm{~s}^{-1}\right)=7 \mathrm{~ms},\left(360^{\circ}\right)^{*}\left(7 \mathrm{~s}^{-1}\right)^{*}$ $\left(7^{*} 10 \mathrm{~ms}\right) \sim 18^{\circ}$.

\subsection{The best-fit electrical parameters}

Table 4 presents the least mean square fitted parameters for these groups. The resistance and capacitance of block $2\left(R_{\mathrm{a} 2}\right.$ and $\left.C_{\mathrm{a} 2}\right)$ are larger than that of the other two blocks $\left(R_{\mathrm{a} 1}, C_{\mathrm{a} 1}\right)$ and $\left(R_{\mathrm{r}}, C_{\mathrm{r}}\right)$. The square roots of $1 / L_{\mathrm{a} 1}{ }^{*} C_{\mathrm{a} 1}, 1 / L_{\mathrm{a} 2}{ }^{*} C_{\mathrm{a} 2}$ and $1 / L_{\mathrm{r}}{ }^{*} C_{\mathrm{r}}$ are also shown which are the natural frequencies $\omega_{f}$ of the three blocks. For control of group A, the natural frequencies are $3.15 \omega_{0}, 1.6 \omega_{0}$ and $5.59 \omega_{0}$ in which $\omega_{0}$ is the radial frequency of the heart rate $\left(\mathrm{rad} \mathrm{s}^{-1}\right)$ given in table 1. For group B, smaller natural frequencies of blocks 1 and 2 were found.

When we compressed the kidney, the resistances and the inductances of the second and the third blocks were increased; however, those of the first block were decreased. Besides, the capacitances of the first and the second blocks became larger with a smaller value found for the third block.

The Ang II effect caused different responses. The resistance of the third block increased greatly and its capacitance decreased. However, for the first and the second blocks, their inductances decreased, capacitances increased, but their resistance variations were small. 
Table 4. Parameters of the three-block model for both groups A and B are presented. The parameters give the least mean square fit with the renal impedance amplitude curves for harmonics $1-7$.

\begin{tabular}{|c|c|c|c|c|c|c|c|c|c|c|c|c|}
\hline & \multicolumn{4}{|c|}{ Block 1} & \multicolumn{4}{|c|}{ Block 2} & \multicolumn{4}{|c|}{ Block 3} \\
\hline & ${ }^{\mathrm{a}} R_{\mathrm{a} 1}$ & bLa1 & ${ }^{\mathrm{c}} \mathrm{Ca} 1$ & ${ }^{\mathrm{d}} n_{f}$ & ${ }^{\mathrm{a}} R_{\mathrm{a} 2}$ & ${ }^{\mathrm{b}} \mathrm{La} 2$ & ${ }^{\mathrm{c}} \mathrm{Ca} 2$ & ${ }^{\mathrm{d}} n_{f}$ & ${ }^{\mathrm{a}} \mathrm{Rr}$ & ${ }^{\mathrm{b}} \mathrm{Lr}$ & ${ }^{\mathrm{c}} \mathrm{Cr}$ & ${ }^{\mathrm{d}} n_{f}$ \\
\hline \multicolumn{13}{|l|}{ Group A } \\
\hline Control & 2.53 & 0.402 & 0.013 & 3.15 & 9.26 & 0.402 & 0.051 & 1.60 & 2.93 & 0.223 & 0.008 & 5.59 \\
\hline Compress & 0.98 & 0.221 & 0.027 & 2.94 & 11.80 & 2.568 & 0.086 & 0.48 & 8.26 & 0.354 & 0.004 & 5.89 \\
\hline \multicolumn{13}{|l|}{ Group B } \\
\hline Control & 0.98 & 0.227 & 0.036 & 2.55 & 11.73 & 0.770 & 0.040 & 1.32 & 1.96 & 0.091 & 0.019 & 5.59 \\
\hline Ang II & 0.39 & 0.140 & 0.066 & 2.48 & 11.73 & 0.093 & 0.055 & 3.34 & 5.38 & 0.140 & 0.013 & 5.66 \\
\hline
\end{tabular}

${ }^{\text {a }} R_{\mathrm{a} 1}, R_{\mathrm{a} 2}, R_{\mathrm{r}}$ is equivalent to the hemodynamic resistance in $10^{5} \mathrm{dyn} \mathrm{s} \mathrm{cm}^{-5}$.

${ }^{\mathrm{b}} L_{\mathrm{a} 1}, L_{\mathrm{a} 2}, L_{\mathrm{r}}$ is equivalent to the hemodynamic inertance in $10^{4} \mathrm{dyn}^{-5}$.

${ }^{\mathrm{c}} C_{\mathrm{a} 1}, C_{\mathrm{a} 2}, C_{\mathrm{r}}$ is equivalent to the hemodynamic compliance in $\mathrm{cm}^{5} /\left(10^{6} \mathrm{dyn}\right)$.

${ }^{\mathrm{d}}$ The square roots of $1 / L_{\mathrm{a} 1}{ }^{*} C_{\mathrm{a} 1}, 1 / L_{\mathrm{a} 2}{ }^{*} C_{\mathrm{a} 2}$ and $1 / L_{\mathrm{r}}{ }^{*} C_{\mathrm{r}}$ are calculated as the natural frequencies $\omega_{f}$ of the three blocks.

${ }^{\mathrm{e}} \omega_{f}=n_{f} \omega_{0}$ in which $\omega_{0}$ is the radial frequency of heart rate given in table 1.

\section{Discussion}

In this report, the characteristic patterns of renal impedance profiles have been studied. A three-block electrical model was built and the electrical parameters were obtained by least mean square fitting of the characteristic impedance profiles. Several important results have been presented.

\subsection{Good matching curves}

Our model provides nearly perfectly matching curves with the amplitude profiles of renal impedance by the least mean square fitted parameters over second harmonics. It also gives phase curves with a similar trend to the experimental phase profiles but with about $20^{\circ}$ discrepancies. Considering the extra $7 \mathrm{~cm}$ travel distance for the pressure wave to go from the measuring point (the intravenous catheter tip) to the pressure transducer, a $20^{\circ}$ delay is reasonable for a pressure wave velocity of $10 \mathrm{~m} \mathrm{~s}^{-1}$. We tried other electrical models with different $R, L, C$ combinations; however, the series connected Windkessels (with inductance) electrical model presented here was the only one to fit the characteristic renal impedance profiles for both amplitude and phase.

\subsection{Classification of renal blood vessels}

From the calculated parameters (the electrical resistance, inductance and capacitance), the three construction blocks simulate distinct physical properties of renal blood vessels (hemodynamic resistance $R_{\text {vas }}$, inertance $L_{\text {vas }}$ and compliance $C_{\text {vas }}$ ) and can be identified as different parts of the renal vascular system. The parameters indicate that the renal artery, the afferent arteriole and the efferent arteriole should belong to different blocks. According to Guyton (1991), the largest resistance posits at the small renal arteries and the afferent arteriole, which is responsible for about $60 \%$ of pressure drop in the renal system. The blood pressure drops from $100 \mathrm{mmHg}$ (the arcuate artery) to $45 \mathrm{mmHg}$ (glomerulus). Therefore, the block with the largest resistance is related to the small arteries plus the afferent arteriole. There are two smaller resistance blocks; the one with larger capacitance implying larger compliance is 
identified as the more elastic renal artery (smaller elastic modulus), and the other block with smaller capacitance is identified as the residual of the kidney which is mainly the efferent arteriole plus the post glomerular capillary structures. The largest capacitance value observed at the small arteries plus the afferent arteriole block may be explained by the fact that the total cross section of these blood vessels is larger than the cross section of the renal artery and their elasticity is greater than that of the capillaries.

Detailed descriptions of hemodynamic resistance $R_{\mathrm{vas}}$, inertance $L_{\mathrm{vas}}$ and compliance $C_{\text {vas }}$ may be found in Milnor's textbook (1989). Roughly, $R_{\mathrm{vas}}$ is related to viscous force; $L_{\mathrm{vas}}$ comes from inertial forces; and these are inversely proportional to the radius of the blood vessels. $C_{\text {vas }}$ deals with the elasticity of blood vessels. It is proportional to the radius but inversely proportional to the wall thickness as well as the elastic modulus of a blood vessel.

It has to be pointed out that the clear cutoff line between the blocks is not as yet well defined; however, it will not change our main inferences if the interlobar arteries are classified into the first block or the glomerular capillaries are classified into the third block, although more detailed information is needed to be sure which block these arteries really belong to.

\subsection{Fidelity of renal blood vessel classifications}

The fidelity of the above classification is further confirmed by the compressing kidney effect and the Ang II effect.

4.3.1. The compressed kidney effect. When we compressed the kidney, the arterioles as well as the capillaries collapsed; flow through the kidney is difficult meaning increased resistance and inertance (as a consequence of a smaller vascular cross section and increasing viscosity) of the affected tissues according to the properties of $R_{\mathrm{vas}}, L_{\mathrm{vas}}$ and $C_{v a s}$ described in section 4.2 (Milnor 1989). Increased resistance and inductance of the second (the small arteries plus the afferent arteriole) and the third blocks (the residual of the kidney) was observed as was expected. In contrast, the untouched renal artery enlarged by backward flow gave smaller resistance $R_{\mathrm{a} 1}$ and smaller inductance $\left(L_{\mathrm{a} 1}\right)$ during kidney compressing. Compliance responses were more complicated though; increasing compliances in the first and the second blocks but decreasing in the third block were seen. Compliance varies with relative blood vessel wall thickness, vessel radius and wall elasticity (Milnor 1989). Compliance should drop when vessel radius is reduced as was seen during the compression effect of the third block. However, the compliance effect on the second block is difficult to evaluate because it is difficult to predicate the wall thickness changes of the elastic small arteries in this situation. In addition, as only part of the kidney could be compressed this allowed for the untouched elastic small arteries to become enlarged by backward blood flow.

4.3.2. Ang II effect. The Ang II effect caused different responses in the blocks. Here, increasing resistance and inductance but decreasing compliance was only seen in the third block. For the first and the second blocks, inductances were decreased and compliances were increased but resistances varied little. This indicates that the blood vessels of the third block were the most constricted. From this it can be inferred that the efferent arteriole plus the post glomerular capillary structures were the main target of Ang II, the renal artery and the small arteries plus the afferent arteriole were less affected. Though there is some controversy about Ang II's effect at the afferent arteriole site (Ichikawa and Harris 1991) perhaps related to dosage or increase of blood pressure, our finding concurs with Guyton's textbook (1991) which declares that the efferent arteriole is much more sensitive to Ang II than the afferent arteriole. Similar Ang II effects were reported by Gross et al (1976). They found that 
Ang II would decrease renal compliance but there was no change in GFR on perfused isolated kidneys of dogs.

\subsection{Comparison with two-block model}

For comparison, a two-block model with similar structure was also tested (data not shown). The simplified two-block model still has a good fit to the fourth harmonic of the impedance profiles. One block with smaller resistance and smaller capacitance with its natural frequency around the frequency of the third harmonic may represent the renal artery. The other with larger resistance and larger capacitance without a consistent natural frequency may represent the kidney. The two-block model loses fidelity at higher frequencies and smears the oscillating nature of the kidney because the physical properties of small arteries and large arterioles are blunted in the two-block model; therefore the model has much less analytical use than the three-block model.

When compressing kidneys or infusing them with Ang II, the changes non-specifically increased resistance and decreased the capacitance of both blocks. The two-block model was unable to indicate exact locations.

\subsection{The best-fit parameters are close to the analytic solutions}

For the three-block model, there are only eight independent parameters in nine (the sum of the three resistance parameters should be equal to the dc resistance measured). However, there are a total of 15 impedance equations to be solved (eight for amplitude profile and seven for phase profile) if the seven harmonics profiles are fitted. The fitting is without extra degrees of freedom, so the best-fit parameters are close to the analytic solutions of the impedance equations. It is highly likely that a model represents the proper physical properties of a studied system if a set of parameters has good fit over seven harmonics for both amplitude and phase angle profiles.

\subsection{The influence from mean blood pressure changes}

In this study, both stimuli caused similar small extent changes on the mean blood pressure but with different impedance effects. It can then be inferred that blood pressure may have little influence on impedance responses.

The three-block model was proved to have good resolvability in indicating locations where varied physical properties change renal impedance. The study shows that impedance does give more information than dc resistance and furthermore our model 'sees' more detailed physical properties of the renal vascular system than just the pure numbers of the impedance. It may help analyze influences on renal circulation due to structural differences between pre-/post glomerular impedance such as in the case of hypertensive SHR rats (Kett et al 2001, Yamamoto et al 2001). The relative work is presently being studied.

\section{Conclusion}

The above discussions strongly suggested that our three-block model represents the proper physical properties of the renal vascular system. The control spectra of both groups show similar patterns. There is an impedance maximum point at the third harmonic and flow leads 
pressure for all harmonics. Both groups present clear and consistent physical properties of the three constructed blocks from the least mean square fitted parameters.

This model described renal impedance characteristics well. It provides useful hints on the physical properties of the renal vascular system and distinguishes possible physiologically affected locations during functional disturbances. Since the improvements in ultrasound Doppler systems have now made it possible to measure continuous non-invasive renal blood flow, our method has potential to be developed as a non-invasive clinical analysis tool.

\section{Appendix}

The impedance equations for the three-block model in figure 2.

$$
Z(\omega)=Z_{\mathrm{a} 1}(\omega)+Z_{\mathrm{a} 2}(\omega)+Z_{\mathrm{r}}(\omega)
$$

in which

$$
\begin{aligned}
& Z_{\mathrm{a} 1}(\omega)=\frac{R_{\mathrm{a} 1}+j \omega L_{\mathrm{a} 1}}{\left(1-\omega^{2} L_{\mathrm{a} 1} C_{\mathrm{a} 1}\right)+j \omega R_{\mathrm{a} 1} C_{a 1}} \\
& Z_{\mathrm{a} 2}(\omega)=\frac{R_{\mathrm{a} 2}+j \omega L_{\mathrm{a} 2}}{\left(1-\omega^{2} L_{\mathrm{a} 2} C_{\mathrm{a} 2}\right)+j \omega R_{\mathrm{a} 2} C_{a 2}} \\
& Z_{\mathrm{r}}(\omega)=\frac{R_{\mathrm{r}}+j \omega L_{\mathrm{r}}}{\left(1-\omega^{2} L_{\mathrm{r}} C_{\mathrm{r}}\right)+j \omega R_{\mathrm{r}} C_{\mathrm{r}}} .
\end{aligned}
$$

Let

$$
\begin{aligned}
& A_{\mathrm{a} 1}=\left(1-\omega^{2} L_{\mathrm{a} 1} C_{\mathrm{a} 1}\right), \quad A_{\mathrm{a} 2}=\left(1-\omega^{2} L_{\mathrm{a} 2} C_{\mathrm{a} 2}\right), \quad A_{\mathrm{r}}=\left(1-\omega^{2} L_{\mathrm{r}} C_{\mathrm{r}}\right) \\
& N_{\mathrm{a} 1}=A_{\mathrm{a} 1}^{2}+\omega^{2} R_{\mathrm{a} 1}^{2} C_{\mathrm{a} 1}^{2}, \quad N_{\mathrm{a} 2}=A_{\mathrm{a} 2}^{2}+\omega^{2} R_{\mathrm{a} 2}^{2} C_{\mathrm{a} 2}^{2}, \quad N_{\mathrm{r}}=A_{\mathrm{r}}^{2}+\omega^{2} R_{\mathrm{r}}^{2} C_{\mathrm{r}}^{2} \\
& Z(\omega)=\left(\frac{R_{\mathrm{a} 1}}{N_{\mathrm{a} 1}}+\frac{R_{\mathrm{a} 2}}{N_{\mathrm{a} 2}}+\frac{R_{\mathrm{r}}}{N_{\mathrm{r}}}\right)+j \omega\left(\frac{L_{\mathrm{a} 1} A_{\mathrm{a} 1}-R_{\mathrm{a} 1}^{2} C_{\mathrm{a} 1}}{N_{\mathrm{a} 1}}+\frac{L_{\mathrm{a} 2} A_{\mathrm{a} 2}-R_{\mathrm{a} 2}^{2} C_{\mathrm{a} 2}}{N_{\mathrm{a} 2}}+\frac{L_{\mathrm{r}} A_{\mathrm{r}}-R_{\mathrm{r}}^{2} C_{\mathrm{r}}}{N_{\mathrm{r}}}\right) \\
& X(\omega)=\left(\frac{R_{\mathrm{a} 1}}{N_{\mathrm{a} 1}}+\frac{R_{\mathrm{a} 2}}{N_{\mathrm{a} 2}}+\frac{R_{\mathrm{r}}}{N_{\mathrm{r}}}\right) \\
& Y(\omega)=\omega\left(\frac{L_{\mathrm{a} 1} A_{\mathrm{a} 1}-R_{\mathrm{a} 1}^{2} C_{\mathrm{a} 1}}{N_{\mathrm{a} 1}}+\frac{L_{\mathrm{a} 2} A_{\mathrm{a} 2}-R_{\mathrm{a} 2}^{2} C_{\mathrm{a} 2}}{N_{\mathrm{a} 2}}+\frac{L_{\mathrm{r}} A_{\mathrm{r}}-R_{\mathrm{r}}^{2} C_{\mathrm{r}}}{N_{\mathrm{r}}}\right) .
\end{aligned}
$$

The impedance amplitude $A_{Z}(\omega)=|Z(\omega)|=\sqrt{X(\omega)^{2}+Y(\omega)^{2}}$; the impedance phase $\theta_{Z}(\omega)=\tan ^{-1}\left(\frac{Y(\omega)}{X(\omega)}\right)$, in which the radial frequency $\omega=2 \pi f ; f$ is the frequency in Hz.

\section{References}

Czosnyka M, Richards H, Pickard J D, Harris N and Iyer V 1994 Frequency-dependent properties of arterial blood transport-an experimental study in anesthetized rabbits Ultrasound Med. Biol. 20 391-9

Giller C A, Ratcliff B, Berger B and Giller A 1996 An impedance index in normal subjects and in subarachnoid hemorrhage Ultrasound Med. Biol. 22 373-82

Gosling R G and King D H 1974 Arterial assessment by Doppler shift ultrasound Proc. R. Soc. Med. $67447-9$

Gross D R, Pimmel R L, Tsai M J and Hamlin R L 1976 Effects of angiotensin and prostaglandin A2 in perfused isolated kidneys of dogs Am. J. Vet. Res. 37 673-80

Guyton A C 1991 Formation of urin by the kidney: 1. Renal blood flow, glomerular filtration, and their control Textbook of Medical Physiology ed M J Wonsiewicz (Philadelphia: WB Saunders Company) pp 286-97 
Handa N, Fukunaga R, Uehara A, Etani H, Yoneda S, Kimura K and Kamada T 1986 Echo-Doppler velocimeter in the diagnosis of hypertensive patients: the renal artery Doppler technique Ultrasound Med. Biol. 12 945-52

Hill A A, Surat D R, Cobbold R S C, Langille B L, Mo L Y L and Adamson S L 1995 A wave transmission model of the umbilicoplacental circulation based on hemodynamic measurements in sheep Am. J. Physiol. 269 (Regul. Integr. Comp. Physiol. 38) R1267-78

Ichikawa I and Harris R C 1991 Angiotensin actions in the kidney: renewed insight into the old hormone Kidney Int. 40 583-96

Jan M Y, Hsiu H, Hsu T L, Wang Lin Y Y and Wang W K 2000 The impedance of pulsatile microcirculation in relation to hypertension IEEE Eng. Med. Biol. Mag. May/June 106-11

Karadeniz T, Topsakal M, Eksioglu A, Ariman A and Basak D 1996 Renal hemodynamics in patients with obstructive uropathy evaluated by color Doppler sonography Eur. Urol. 29 298-301

Kett M M, Bergstrom G, Alcorn D, Bertram J F and Anderson W P 2001 Renal vascular resistance properties and glomerular protection in early established SHR hypertension J. Hypertension 19 1505-151

Merkus J W S, Hoitsma A J, Van Asten W N J C, Koene R A P and Skotnicki S H 1994 Doppler spectrum analysis to diagnose rejection during post transplant acute renal failure Transplantation $\mathbf{5 8} 570-6$

Milnor W R 1989 Vascular impedance Hemodynamics ed N Collins (Baltimore: Williams \& Wilkins) pp 167-203

Mo L Y L, Bascom P A S, Ritchie K and McCowan L M E 1988 A transmission line modeling approach to the interpretation of uterine Doppler waveforms Ultrasound Med. Biol. 14 365-76

Noordergraaf A 1969 Hemodynamics Biological Engineering ed H P Schwan (New York: McGraw Hill) pp 391-545

Pozniak M A, Kelcz F, Stratta R J and Oberley T D 1988 Extraneous factors affecting resistive index Invest. Radiol. 23 899-904

Raines J K, Jaffrin M Y and Shapiro A H 1974 A computer simulation of arterial dynamics in the human leg J. Biomech. 7 77-91

Rothe C F and Nash F D 1967 Renal arterial compliance and conductance measurement using on-line self-adaptive analog computation of model parameters Med. Biol. Eng. 6 53-69

Salgado O, Gracia R, Henriquez C, Rodriguez-Iturbe B and Tahan J E 1997 Renal duplex ultrasonography in the diagnosis and follow up of a case of accelerated transplant rejection treated with OKT3 J. Ultrasound Med. 16 699-702

Spencer M P and Denison A B Jr 1963 Pulsatile blood flow in the vascular system Handbook of Physiology: Circulation section 2, vol 2 ed W F Hamilton and P Dow (Washington, DC: American Physiology Society) pp 839-64

Veglio F, Frascisco M, Melchio R, Provera E, Rabbia F, Oliva S and Chiandussi L 1995 Assessment of renal resistance index after captopril test by Doppler in essential and renal vascular hypertension Kidney Int. 48 1611-6

Warshauer D M, Taylor K J, Bia M J, Marks W H, Weltin G G, Rigsby C M, True L D and Lorber M I 1988 Unusual causes of increased vascular impedance in renal transplants: duplex Doppler evaluation Radiology 169 367-70

Yamamoto T, Tomura Y, Tanaka H and Kajiya F 2001 In vivo visualization of characteristics of renal microcirculation in hypertensive and diabetic rats Am. J. Physiol. (Renal Physiol.) 281 F571-7 